\title{
Selection of a SARS-CoV-2 Surrogate for Use in Surface Disinfection Efficacy Studies with Chlorine and Antimicrobial Surfaces
}

\author{
Gabrielle M. String,* Mitchell R. White, David M. Gute, Elke Mühlberger, and Daniele S. Lantagne \\ Cite This: https://doi.org/10.1021/acs.estlett.1c00593 \\ Read Online
}

ABSTRACT: Initial recommendations for surface disinfection to prevent SARS-CoV-2 transmission were developed using previous evidence from potential surrogates. To the best of our knowledge, no appropriate surrogate for SARS-CoV-2 has been identified or confirmed for chlorine and antimicrobial surface disinfection. We completed a study to evaluate the efficacy of two hypothesized antimicrobial surfaces, and four chlorine solutions on nonporous and porous surfaces, against SARS-CoV-2 and three potential SARSCoV-2 surrogates [coronavirus mouse hepatitis virus (MHV) and bacteriophages Phi6 and MS2], to identify a BSL-1 or BSL-2 virus to use in future studies. We found SARS-CoV-2 can be reduced $>4$ $\log _{10}$ on porous and nonporous surfaces within $30 \mathrm{~s}$ upon exposure
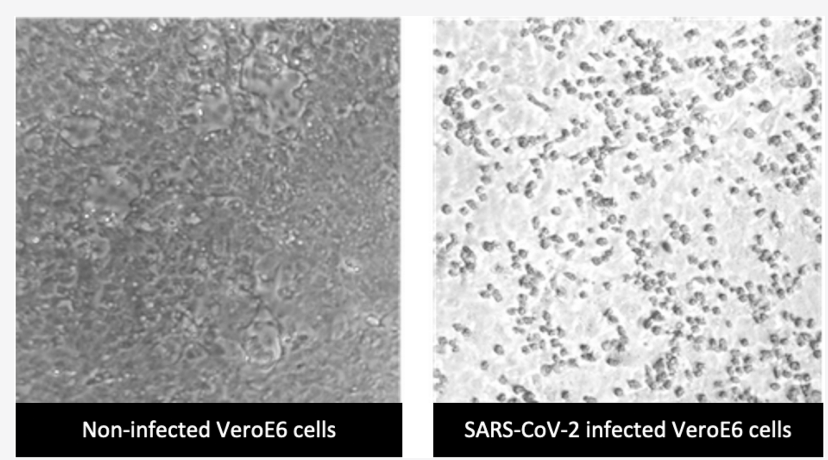
to $0.5 \% \mathrm{NaOCl}$. The results indicate coronavirus MHV-GFP is inactivated faster than SARS-CoV-2 (MHV-GFP $\geq 6.08 \log _{10}$; SARS-CoV-2 $=0.66 \log _{10}$ at $30 \mathrm{~s}$ with $0.05 \% \mathrm{NaOCl}$ on steel) and MS2 is inactivated more slowly. Phi6 is inactivated like SARS-CoV-2, and we propose Phi6 as a slightly conservative surrogate for SARS-CoV-2 chlorine disinfection. Additionally, disinfection of bacteriophages on wood was challenging, and exposure to antimicrobial surfaces had no disinfection efficacy as tested. We recommend using $0.5 \%$ chlorine on surfaces for a minimum of $30 \mathrm{~s}$ of contact to disinfect SARS-CoV-2 and recommend additional research on Phi6 disinfection with varied surfaces and conditions.

\section{INTRODUCTION}

SARS-CoV-2 is an enveloped, positive sense, single-stranded RNA virus from the Coronoviridae family that causes COVID19. ${ }^{1}$ The primary SARS-CoV-2 transmission route is person to person via inhalation; as such, primary infection prevention and control measures include masking and physical distancing. ${ }^{2}$ During outbreaks, disinfecting surfaces in households, community structures, and healthcare facilities is commonly performed to interrupt disease transmission. ${ }^{3,4}$ Although fomites are not a primary transmission route, to prevent SARS-CoV-2 transmission the World Health Organization and U.S. Centers for Disease Control and Prevention (CDC) recommend cleaning surfaces with soap and water and then using an approved disinfectant or an appropriate concentration of chlorine. 5,6

A recent systematic review ${ }^{7}$ identified 10 surface disinfection tests conducted with SARS-CoV-2 and 15 conducted with potential surrogates and found sunlight, ultraviolet light, ethanol, hydrogen peroxide, and chlorine can reduce SARSCoV-2 or surrogates by $\geq 99.9 \%$ on surfaces. Surrogates can be used as a model for highly infectious pathogens, as largevolume testing can be conducted with a surrogate and confirmed with small-volume infectious pathogen testing. ${ }^{8}$ To the best of our knowledge, no appropriate surrogate has been identified or confirmed for chlorine surface disinfection with SARS-CoV-2.
Our goal was to identify a BSL-1 or BSL-2 surrogate for SARS-CoV-2 surface disinfection studies, for chlorination and antimicrobial surfaces. First, we evaluated the efficacy of chlorine disinfection against SARS-CoV-2 on nonporous and porous surfaces. Then, we replicated experiments to evaluate three potential surrogates. Lastly, we tested the efficacy of two potentially antimicrobial materials against SARS-CoV-2 and the three potential surrogates.

\section{MATERIALS AND METHODS}

This study was completed by selecting surrogates, preparing materials, conducting experimental protocols, and analyzing data. More details about the methods can be found in the Supporting Information.

Surrogate Selection. Surrogates were selected using the framework of Sinclair et al. ${ }^{8}$ to identify candidates on the basis of safety and resistance to chlorine disinfection. A primary goal for the research was to identify a BSL-1 or BSL-2 surrogate, for

Received: July 26, 2021

Revised: September 28, 2021

Accepted: September 29, 2021 
use in future studies. We selected murine hepatitis virus (MHV), Phi6, and MS2 for the study. MHV is a member of the Coronaviridae family ${ }^{9}$ and shares characteristics with SARS-CoV-2 (Table S1). As a BSL-2 virus, MHV has been used as a SARS-CoV-2 surrogate for disinfection ${ }^{7}$ and was found to be resistant to disinfection by $0.06 \% \mathrm{NaOCl}$ on stainless steel. ${ }^{10}$ We utilized MHV expressing green fluorescent protein (MHV-GFP). Recombinant coronaviruses expressing reporter proteins, such as GFP, are widely used for antiviral activity assays. Because the virally encoded reporter protein is not incorporated into the viral particles, it does not alter the structure of the virions or their physicochemical properties. ${ }^{11,12}$ Therefore, it is assumed that MHV-GFP has the same susceptibility to disinfection as wild-type MHV. We selected two BSL-1 bacteriophages, Phi6 ${ }^{13}$ and MS2. ${ }^{14}$ Phi6 is enveloped like SARS-CoV-2 (Table S1), has been used as a SARS-CoV-2 surrogate for disinfection, ${ }^{7,15}$ and is an appropriate surrogate for $\mathrm{H} 5 \mathrm{~N} 1$ avian influenza virus and Ebola virus for chlorine disinfection. ${ }^{16,17}$ While MS2 is nonenveloped, it is a positive sense single-stranded RNA virus like SARS-CoV-2 (Table S1) and has been used as a surrogate of enteric viruses, ${ }^{18}$ and the efficacy of free chlorine disinfection on surfaces has been well-documented. ${ }^{17-19}$

Preparation of Materials. Surface Carriers. Five surfaces (steel, plastic, wood, copper, and Supertowel) were tested in total. Three surfaces, representing a range of materials found in low-resource contexts, were chosen for chlorine disinfection, including nonporous (stainless steel and polypropylene plastic) and porous (wood) materials. ${ }^{20}$ Copper was chosen as an antimicrobial surface because of its use in brass taps, a hightouch surface during water collection in some low-resource contexts, and previous literature documenting its antimicrobial properties. $^{21}$ Supertowel (Real Relief Way, Kolding, Denmark), a reusable microfiber handwashing towel with a permanently bonded antimicrobial layer, was chosen as it is intended for use where handwashing materials (e.g., soap and water) are scarce, and previous research on antimicrobial towels has recovered viable bacteria from the surface after use. $^{22}$ Surface carriers were prepared from a virgin stock of each material (see the Supporting Information); $1 \mathrm{~cm}$ diameter disks were used for stainless steel, plastic, wood, and copper, and $0.88 \mathrm{~cm}^{2}$ squares (the same surface area as disks) were used for Supertowel. Carriers were appropriately sterilized before use.

Chlorine Solutions. Two hours before testing, $\mathrm{pH}$-stabilized sodium hypochlorite chlorine solution concentrations were confirmed to be within $10 \%$ of those of target solutions: $0.01 \%$ (100 mg/L), $0.05 \%$ (500 mg/L), $0.1 \%$ (1000 mg/L), or $0.5 \%$ chlorine $(5000 \mathrm{mg} / \mathrm{L})$, using iodometric titration (Hach Co.), as detailed in the Supporting Information. On the basis of previous testing and results, the chlorine demand of the virus/ phage stocks should represent $<0.01 \%$ of the minimum available chlorine ${ }^{17}$ and stock solutions should not degrade within the testing window. ${ }^{23}$

Viruses and Cell Lines. Mouse cell lines L-929 (ATCCCCL-1) and 17CL-1 (provided by V. Thiel, University of Bern, Bern, Switzerland) were maintained in Eagle's minimum essential medium (EMEM; Corning, 10-009-CV) supplemented with $10 \%$ fetal bovine serum (FBS) and $100 \mu \mathrm{g} /$ $\mathrm{mL}$ primocin (Invivogen, ant-pm-2). Monkey kidney cell line Vero E6 (ATCCCRL-1586) was maintained in Dulbecco's modified Eagle's medium (DMEM; Lonza, catalog no. 12$614 \mathrm{~F}$ ) supplemented with $1 \%$ L-glutamine, $10 \%$ fetal bovine serum (FBS), and $100 \mu \mathrm{g} / \mathrm{mL}$ primocin. MHV-GFP provided by V. Thiel was propagated in either L-929 or 17CL-1 cells in EMEM supplemented with $2 \%$ FBS and $100 \mu \mathrm{g} / \mathrm{mL}$ primocin. SARS-CoV-2 (isolate USA_WA1/2020) was provided by CDC's Principal Investigator N. Thornburg and the World Reference Center for Emerging Viruses and Arboviruses (WRCEVA) and propagated in VeroE6 cells in DMEM supplemented with $1 \%$ L-glutamine, $2 \%$ FBS, and $100 \mu \mathrm{g} / \mathrm{mL}$ primocin. $^{24}$ Viral titers were determined by tissue culture infectious dose $\left(\mathrm{TCID}_{50}\right)$ assays.

Bacteriophages. Bacteriophages were propagated via the double agar overlay method ${ }^{25}$ using Nutrient Broth Yeast (NBY) containing a $\mathrm{CaCl}_{2} / \mathrm{D}$-glucose solution for preparation of media and agars. Phi6 (HER\#102) was propagated in Pseudomonas syringae (HER\#1102), and MS2 (ATCC15597B1) was propagated in Escherichia coli (ATCC15597) (see the Supporting Information).

Experimental Protocol. Test methods were adapted from the ASTM International Method for Standard Quantitative Disk Carrier Test for testing the virucidal activity of chemicals. ${ }^{26}$ All tests, including positive and negative controls, were completed in triplicate. Samples were sacrificial, as different disks were inoculated for each exposure time point. Before testing, we ensured sodium thiosulfate solutions did not have a cytotoxic effect on host or virus/phage organisms.

Chlorine Disinfection. In a biosafety cabinet, $10 \mu \mathrm{L}$ of virus/phage was pipetted onto the center of steel and plastic carriers; $50 \mu \mathrm{L}$ of virus/phage was pipetted onto wood carriers to assist recovery from the thicker, porous surface. After samples had been dried for $1 \mathrm{~h}, 50 \mu \mathrm{L}$ of a chlorine solution was applied to steel and plastic carriers $(250 \mu \mathrm{L}$ to wood carriers). After exposure for $0.5,1,3$, or $5 \mathrm{~min}, 950 \mu \mathrm{L}$ of $0.25 \%$ sodium thiosulfate was applied to steel and plastic carriers ( $700 \mu \mathrm{L}$ to wood carriers) to neutralize the chlorine. On positive controls, viruses/phage were added as described above without chlorine. On negative controls virus dilution buffer (VDB), a sodium phosphate salt solution, was added in place of virus/phage. On positive and negative controls, 1000 $\mu \mathrm{L}$ of sodium thiosulfate was added as an eluent. Samples were vortexed, recovered by pipetting into sterile Eppendorf tubes, and stored at $4{ }^{\circ} \mathrm{C}$ for $\leq 3 \mathrm{~h}$ until quantification.

Disinfection with Antimicrobial Surfaces. In a biosafety cabinet, $10 \mu \mathrm{L}$ of virus/phage was pipetted onto the center of copper carriers, and $50 \mu \mathrm{L}$ of virus/phage was pipetted onto Supertowel carriers (presoaked with PBS) to ensure the sample could be recovered from the absorbent surface. After 1, 5, 10, or $15 \mathrm{~min}$ on the surfaces, $950 \mu \mathrm{L}$ of $0.25 \%$ sodium thiosulfate was applied to copper carriers and $700 \mu \mathrm{L}$ was applied to Supertowel carriers as an eluent. Immediately after sodium thiosulfate was applied, samples were prepared as detailed above, except a Falcon cell scraper was used after vortexing Supertowel samples to squeeze liquid from the towel to increase the volume recovered. Positive and negative controls were prepared as described above.

Virus/Phage Quantification. 17CL-1 (for MHV-GFP titration) or VeroE6 cells (for SARS-CoV-2 titration) were seeded in 96-well tissue culture plates at a density of $1 \times 10^{4}$ cells per well in $180 \mu \mathrm{L}$ of the appropriate cell culture medium containing $2 \%$ FBS. Twenty microliter samples of virus collected from surface carriers with or without chlorine treatment were added to the appropriate cells seeded in the first row of a 96-well plate in quadruplicate followed by 10 -fold dilutions across the plate. Plates were incubated at $37^{\circ} \mathrm{C}$ with 


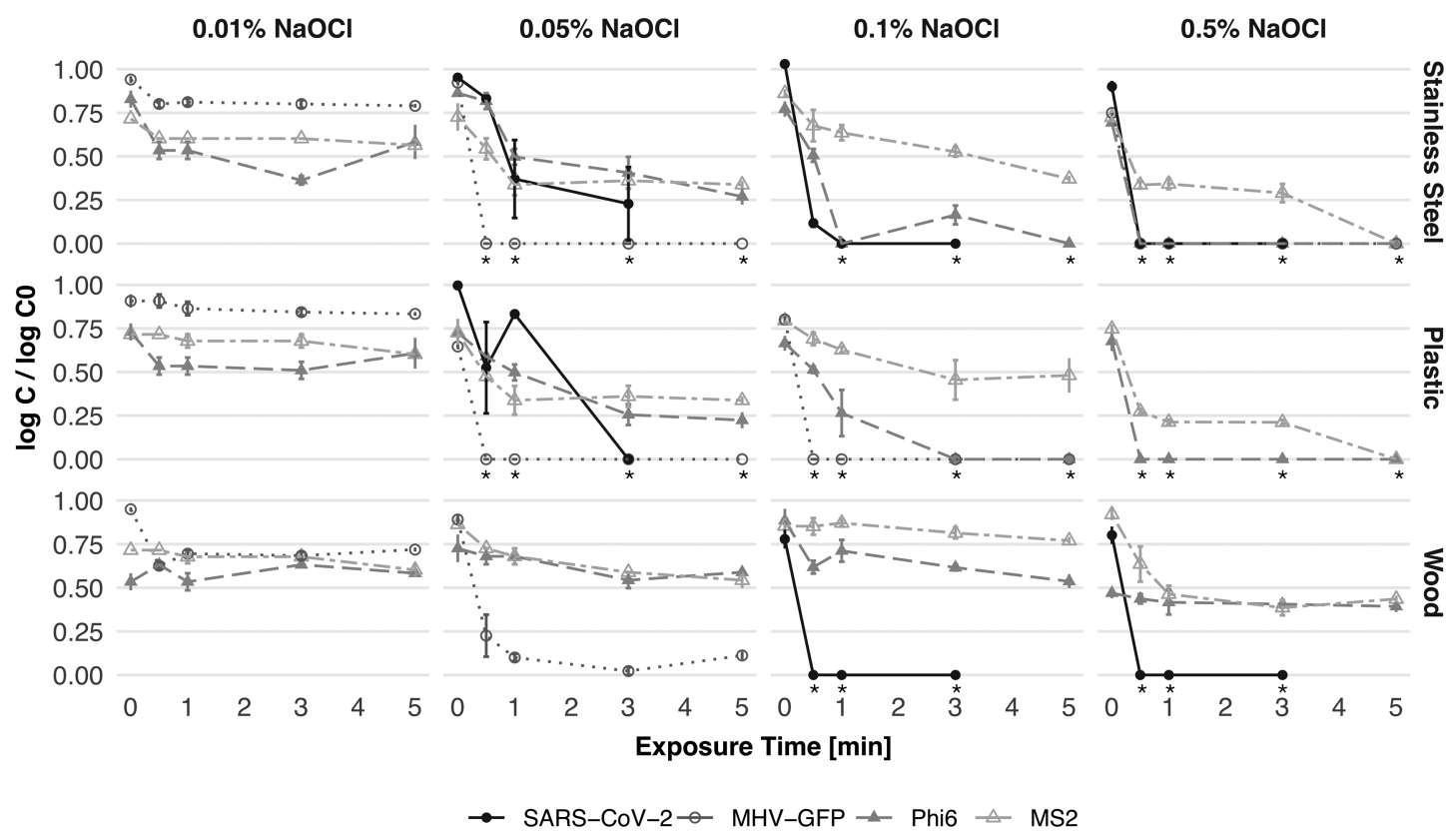

Figure 1. Results of disinfection tests conducted on stainless steel, plastic, and wood surfaces with viruses exposed to $0.01-0.5 \%$ chlorine for 0.5 , 1 , 3 , and 5 min. Data from four viruses (SARS-CoV-2, MHV-GFP, Phi6, and MS2) expressed as the ratio of the $\log _{10}$ concentration recovered from the surface compared to the $\log _{10}$ stock concentration at each exposure time. Titers were initially calculated in either TCID 50 units per milliliter or plaque-forming units per milliliter. Error bars indicate the standard error of the mean of triplicate tests, and asterisks denote nondetects. The limit of detection was $100 \mathrm{TCID}_{50}$ units for SARS-CoV-2, $10 \mathrm{TCID}_{50}$ units for MHV, and $20 \mathrm{PFU} / \mathrm{mL}$ for Phi6 and MS-2.

$5 \% \mathrm{CO}_{2}$ for 3 days (MHV-GFP) or 14 days (SARS-CoV-2) until fluorescent or cytopathic effect readings were consistent. Viral titers were determined using the Spearman and Kärber algorithm. For bacteriophages, appropriate serial dilutions of samples were prepared in Eppendorf tubes containing $900 \mu \mathrm{L}$ of VDB. Samples, including the stock used in each test series, were titrated by spotting $50 \mu \mathrm{L}$, in duplicate, onto $4 \mathrm{~mL}$ of NBY soft agar containing $65 \mu \mathrm{L}$ of the bacterial host culture already poured onto NBY hard agar plates. Plaques were enumerated after plates had been incubated overnight at the appropriate temperature.

Safety. The protocols described above were carried out by two different trained researchers, one at Boston University (BU) and one at Lancon Environmental, LLC, following institutional, state, and federal guidelines. SARS-CoV-2 testing was completed in the BSL-4 facility of the National Emerging Infections Diseases Laboratories at BU following approved SOPs. MHV-GFP testing was completed in a BSL-2 laboratory at $\mathrm{BU}$. To reduce unnecessary exposure to infectious pathogens, select disinfection tests were conducted with SARS-CoV-2 and MHV-GFP in a stepwise fashion informed by results (see the Supporting Information). Tests with bacteriophages were completed in a BSL-2 laboratory at Lancon.

Analysis. Data were entered into Microsoft Excel (Microsoft, Redmond, WA). The limit of detection was $100 \mathrm{TCID}_{50}$ units for SARS-COV-2 and $10 \mathrm{TCID}_{50}$ units for MHV-GFP. Bacteriophage samples with no detected plaques were replaced with 10 plaque-forming units $(\mathrm{PFU}) / \mathrm{mL}$, half the theoretical minimum detection limit, and plates with plaques too numerous to count were replaced with $300 \mathrm{PFU} / \mathrm{mL}$, the theoretical maximum detection limit. Recovery percentages were calculated for each experiment by comparing positive controls $(t=0)$ to inoculate titers for each experiment and surface. $\log _{10}$ reduction values (LRVs) and standard errors were calculated for each sample and averaged over triplicate tests. Lastly, plots of the ratio of the $\log _{10}$ titer recovered from the surface to the $\log _{10}$ titer of the inoculation stock were generated and used to compare reduction of viruses/phages at each time point.

\section{RESULTS AND DISCUSSION}

In total, 146 tests were conducted with chlorine and 32 with antimicrobial surfaces in triplicate (totaling 438 chlorine tests, 96 antimicrobial) and positive (time zero) and negative controls. Average stock concentrations were $3.96 \times 10^{5}$ $\mathrm{TCID}_{50}$ units $/ \mathrm{mL}$ for SARS-CoV-2, $4.12 \times 10^{7} \mathrm{TCID}_{50}$ units/mL for MHV-GFP, $4.65 \times 10^{8} \mathrm{PFU} / \mathrm{mL}$ for Phi6, and $1.01 \times 10^{11} \mathrm{PFU} / \mathrm{mL}$ for MS2. The percent recovery was variable for all viruses and surfaces: $10-95 \%$ for SARS-CoV-2 (wood to plastic), $0.19-99 \%$ for MHV-GFP (plastic to copper), $0.01-17 \%$ for Phi6 (wood to Supertowel), and $0.05-39 \%$ for MS2 (steel to wood) (Tables S2 and S3).

Viruses were minimally reduced after 5 min with $0.01 \%$ $\mathrm{NaOCl}$ (Figure 1 and Table S4). MHV-GFP was reduced $\geq 4.96$ to $\geq 7.08 \log _{10}$ with $0.05 \% \mathrm{NaOCl}$ (Table S4), but SARS-CoV-2 was reduced $4.05-5.58 \log _{10}$ on stainless steel at the conclusion of the test (Figure 1). Exposure to $0.1 \% \mathrm{NaOCl}$ resulted in SARS-CoV-2 reduction of $\geq 4.36$ to $\geq 5.31 \log _{10}$ after $1 \mathrm{~min}$ (wood and stainless steel), Phi6 reduction of $\geq 5.04$ to $\geq 6.01 \log _{10}$ after $5 \mathrm{~min}$ (plastic and stainless steel) (Figure 1), but lower LRVs (3.15-4.93) for MS2 after 5 min (stainless steel and plastic) (Table S4). SARS-CoV-2 was reduced $>4$ LRV with $0.5 \% \mathrm{NaOCl}$ after exposure for $0.5 \mathrm{~min}$ on stainless steel and wood; Phi 6 was reduced $>5$ LRV after $0.5 \mathrm{~min}$, and MS2 was reduced $>7$ LRV after 5 min on stainless steel and plastic (Figure 1). Both Phi6 and MS2 were more resistant than SARS-CoV-2 on wood with minimal reduction after exposure for $5 \mathrm{~min}$ (0.59 LRV for Phi6 and 3.92 LRV for MS2) (Table S4). 

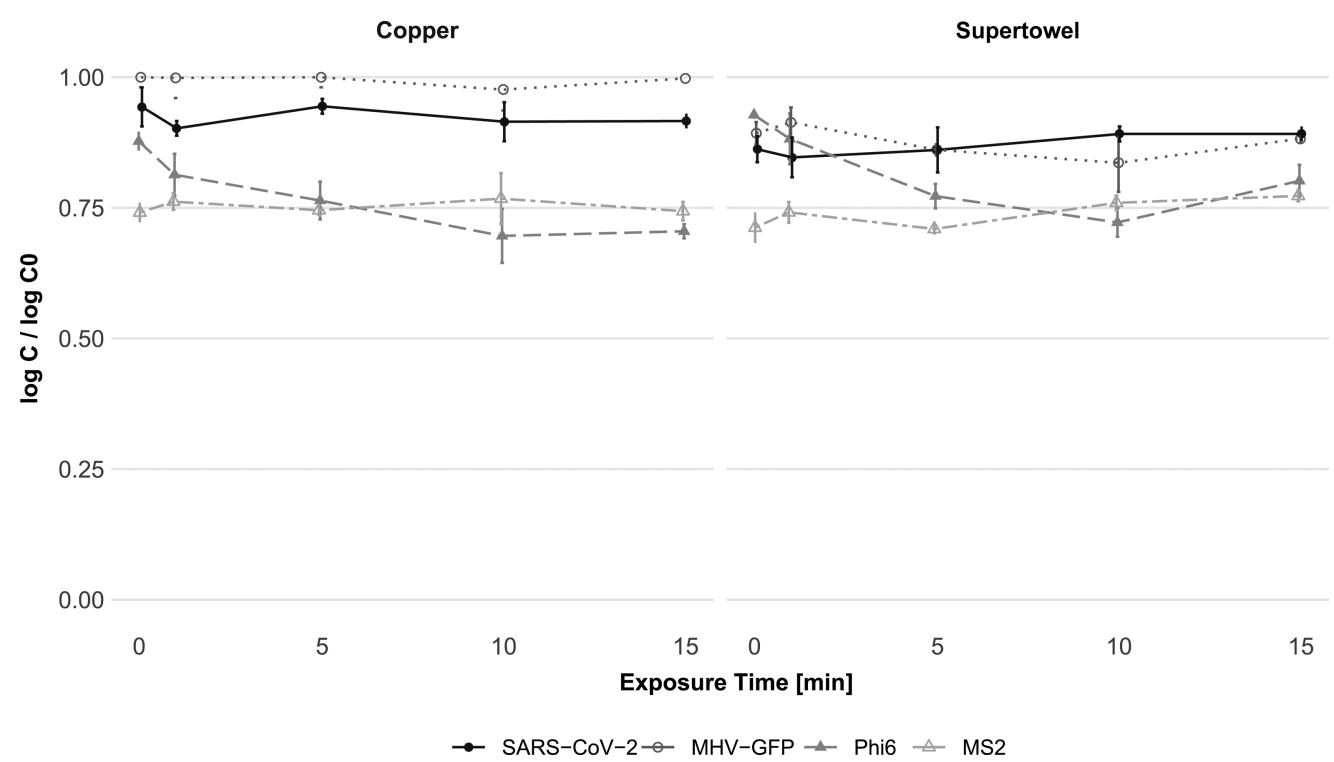

Figure 2. Results of disinfection tests conducted on antimicrobial surfaces copper and Supertowel after exposure for 1, 5, 10, and 15 min. Data from four viruses (SARS-CoV-2, MHV-GFP, Phi6, and MS2) expressed as the ratio of the $\log _{10}$ concentration recovered from the surface compared to the $\log _{10}$ stock concentration at each exposure time. Titers were initially calculated in either $\mathrm{TCID}_{50}$ units per milliliter or plaque-forming units per

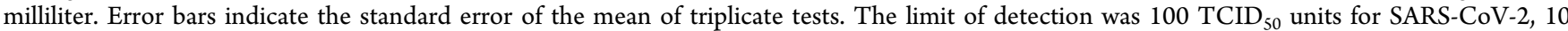
$\mathrm{TCID}_{50}$ units for MHV, and $20 \mathrm{PFU} / \mathrm{mL}$ for Phi6 and MS-2.

The hypothesized antimicrobial surfaces of copper and Supertowel did not inactivate applied viruses within $15 \mathrm{~min}$ of exposure (Figure 2 and Table S5).

Chlorine Is Efficacious against SARS-CoV-2. Overall, the results indicate SARS-CoV-2 can be reduced $>4 \log _{10}$ on porous and nonporous surfaces upon exposure for $1 \mathrm{~min}$ to $0.1 \% \mathrm{NaOCl}$ and for $30 \mathrm{~s}$ to $0.5 \% \mathrm{NaOCl}$, confirming existing recommendations ${ }^{5,6,27}$ and corroborating a recent study reporting $>4.75 \mathrm{LRV}$ after exposure to $0.1 \%$ for $0.5 \mathrm{~min}$ and $0.05 \%$ for $5 \mathrm{~min}^{28}$ The choice to use $0.5 \% \mathrm{NaOCl}$ aligns with recommendations for chlorine disinfection in Ebola outbreaks, ${ }^{29}$ thus enabling use by responders in low-resource settings.

MHV Is a Poor Surrogate for SARS-CoV-2 in Chlorine Disinfection. MHV-GFP was reduced $\geq 7.08 \log _{10}$ on stainless steel and $\geq 4.96 \log _{10}$ on plastic after exposure for $30 \mathrm{~s}$ to $0.05 \% \mathrm{NaOCl}$, while SARS-CoV-2 was reduced 0.67 $\log _{10}$ on steel and $2.64 \log _{10}$ on plastic at the same dose and exposure time. Although MHV has been used as a surrogate for SARS-CoV-2 in disinfection, it is possible chlorine targets MHV-GFP differently than SARS-CoV-2, and further research to elucidate the mechanisms of inactivation is necessary. In particular, the spike proteins of SARS-CoV-2 and MHV bind to different cellular receptors, angiotensin converting enzyme 2 $(\mathrm{ACE} 2)^{30}$ and mouse carcinoembryonic antigen-related cell adhesion molecule 1a (mCEACAM1a), ${ }^{31}$ and the receptor binding domains are located in different regions of the spike proteins, ${ }^{32,33}$ which might affect inactivation mechanisms. For example, it is conceivable that the structure of the MHV receptor binding domain is destroyed with low chlorine concentrations, whereas the SARS-CoV-2 binding domain is more stable. However, this is highly speculative and requires further research.

Phi6 as a Surrogate. We propose Phi6 as a conservative and appropriate BSL-1 surrogate for SARS-CoV-2 in chlorine disinfection as it was inactivated after a longer exposure to $0.1 \% \mathrm{NaOCl}$ than SARS-CoV-2 but in a manner similar to that of SARS-CoV-2 with $0.5 \% \mathrm{NaOCl}$ (steel). Bacteriophages Phi6 and MS2 were both more resistant to disinfection with $0.1 \%$ $\mathrm{NaOCl}$ than SARS-CoV-2 on all three surfaces. However, Phi6 was reduced more after exposure to $0.1 \%$ and $0.5 \% \mathrm{NaOCl}$ than MS2, a trend observed previously. ${ }^{17}$ As a previously proposed model for other viruses, ${ }^{16,17}$ the literature on Phi6 chlorine disinfection efficacy can enhance our understanding of SARS-CoV-2. ${ }^{34-38}$ Furthermore, as Phi6 is a simple-to-use BSL-1 bacteriophage, surface and handwashing efficacy laboratory work can be facilitated. At a minimum, results could inform Ebola and SARS-CoV-2 recommendations and potentially (after confirmation) those for a novel emerging virus.

The Disinfection Efficacy on Wood Varied. Herein, disinfection of bacteriophages was more challenging on wood surfaces than that of coronaviruses. For coronaviruses, MHV was $6.12 \log _{10}$ on wood and $\geq 4.96$ to $\geq 7.08 \log _{10}$ on plastic and steel at $0.05 \% \mathrm{NaOCl}$, and for SARS-CoV-2 reduction by $\geq 4 \log _{10}$ within $1 \mathrm{~min}$ of exposure to $0.1 \%$ and $0.5 \% \mathrm{NaOCl}$ on both steel and wood. For bacteriophages, inactivation was not consistent between nonporous and porous surfaces, with a $\geq 5.15 \log _{10}$ reduction in phage after exposure for $5 \mathrm{~min}$ to $0.5 \% \mathrm{NaOCl}$ on steel and plastic but reduction of $0.59-3.92$ $\log _{10}$ on wood. Surface porosity has reduced the efficacy of disinfection previously, ${ }^{20,39}$ and chlorine has been found to be less efficacious on wood than on nonporous surfaces. ${ }^{40,41}$ Further research is indicated to understand if this variation was due to the surface type inhibiting chlorine efficacy and recovery or differences in pipetting techniques between researchers.

Antimicrobial Surfaces Did Not Reduce Viruses. Exposure to copper and Supertowel has no biocidal effects as tested. We hypothesized copper could have reduced SARSCoV-2 because copper in solution inactivates several viruses. $^{42,43}$ This mechanism of contact killing ${ }^{21}$ was not efficacious in our results, indicating that copper solution biocidal efficacy should not be translated to surfaces. 
Additionally, we tested a 15 min exposure time to mimic the maximum feasible amount of time copper-coated high-touch surfaces might remain undisturbed, but evidence suggests the persistence of SARS-CoV-2 on copper exceeds $4 \mathrm{~h}^{44}$ While previous work has highlighted the efficacy of Supertowel to reduce $E$. coli in use while handwashing, ${ }^{45}$ we found viruses/ phages dried on the towel were not inactivated in a $15 \mathrm{~min}$ exposure period. Further research is necessary to understand the efficacy of Supertowel virus removal in handwashing practice, including after friction and handrubbing with the towel.

Limitations. The following limitations were documented. (1) Testing was completed by two different researchers; variations between laboratory technique may have influenced results in unknown ways. ${ }^{46}$ (2) Although we monitored laboratory temperature and humidity and confirmed chlorine concentrations to be within $10 \%$ of the target, tests among the four organisms were completed at different times. (3) Although we observed similar trends across test conditions, our data set is too small to reliably complete statistical analysis. (4) Recovery was variable, although similar to reported viral recovery rates on other porous and nonporous surfaces. ${ }^{17,39}$ (5) Titers of stock varied between viruses and phages. (6) Copper and Supertowel surfaces were evaluated in ways that may not reflect actual use situations.

Recommendations. On the basis of our results, we conservatively recommend the use of $0.5 \% \mathrm{NaOCl}$ with a minimum of $30 \mathrm{~s}$ of contact to disinfect nonporous and porous surfaces to prevent SARS-CoV-2 transmission in healthcare facilities and high-touch contexts. Future research is needed to understand the impact of soil load, the presence of additional organisms, the chlorine application mode, and the impact of surface type on chlorine disinfection efficacy. We discourage the use of coronavirus MHV-GFP as a surrogate for SARSCoV-2 in chlorine disinfection studies and propose the bacteriophage Phi6 as a model organism. Future work is needed to investigate the chlorine disinfection mechanism of MHV-GFP and SARS-CoV-2 to understand why this promising surrogate is not appropriate. We recommend investigating the impact of humidity and temperature on disinfection efficacy as these parameters can impact Phi6 survivability ${ }^{47}$ and whether Phi6 is an appropriate surrogate for SARS-CoV-2 for other disinfectants (where mechanisms might vary), such as ultraviolet light, simulated sunlight, ethanol, and hydrogen peroxide. ${ }^{48}$ Lastly, we recommend testing the disinfection mechanism of antimicrobial surfaces in practical use.

\section{ASSOCIATED CONTENT}

\section{(5) Supporting Information}

The Supporting Information is available free of charge at https://pubs.acs.org/doi/10.1021/acs.estlett.1c00593.

Details about methods and additional data, including characteristics of tested viruses/phages (Table S1), percent recovery calculations for viruses/phages stratified by surface (Tables S2 and S3), and $\log _{10}$ reduction values for each virus/phage stratified by disinfectant (Tables S4 and S5) (PDF)

\section{AUTHOR INFORMATION}

\section{Corresponding Author}

Gabrielle M. String - Lancon Environmental, LLC, Cambridge, Massachusetts 02140, United States; Civil and Environmental Engineering, Tufts University School of Engineering, Medford, Massachusetts 02155, United States; (1) orcid.org/0000-0002-0266-923X;

Email: gabrielle.string@tufts.edu

\section{Authors}

Mitchell R. White - Department of Microbiology, Boston University School of Medicine, Boston, Massachusetts 02118, United States; National Emerging Infectious Diseases Laboratories, Boston University, Boston, Massachusetts 02118, United States

David M. Gute - Civil and Environmental Engineering, Tufts University School of Engineering, Medford, Massachusetts 02155, United States

Elke Mühlberger - Department of Microbiology, Boston University School of Medicine, Boston, Massachusetts 02118, United States; National Emerging Infectious Diseases Laboratories, Boston University, Boston, Massachusetts 02118, United States

Daniele S. Lantagne - Lancon Environmental, LLC, Cambridge, Massachusetts 02140, United States; () orcid.org/0000-0001-6594-0261

Complete contact information is available at:

https://pubs.acs.org/10.1021/acs.estlett.1c00593

\section{Notes}

The authors declare no competing financial interest.

\section{ACKNOWLEDGMENTS}

This study is made possible by the generous support of the American people through the U.S. Agency for International Development (USAID). The authors thank CDC's Principal Investigator Natalie Thornburg and the World Reference Center for Emerging Viruses and Arboviruses (WRCEVA) for sharing SARS-CoV-2, Volker Thiel (University of Bern) for sharing MHV-GFP and 17CL-1 cells, and Prof. Sylvain Moineau of the University of Laval (Québec, QC) for Phi6. The authors are grateful to Yarmina Kamal for laboratory assistance at Lancon Environmental, LLC, and Adam Hume and Judith Olejnik for BSL-4 laboratory assistance at the NEIDL at BU. The authors thank RealRelief for providing the Supertowels, Travis Yates and Mike Eubanks for making the surface carriers, and Claire Anderson and Marlene Wolfe for assistance with $\mathrm{Phi} 6$ propagation protocols.

\section{REFERENCES}

(1) Zheng, J. SARS-CoV-2: An Emerging Coronavirus That Causes a Global Threat. Int. J. Biol. Sci. 2020, 16 (10), 1678-1685.

(2) Transmission of SARS-CoV-2: implications for infection prevention precautions. https://www.who.int/news-room/ commentaries/detail/transmission-of-sars-cov-2-implications-forinfection-prevention-precautions (accessed 2021-03-09).

(3) Howard, G.; Bartram, J.; Brocklehurst, C.; Colford, J. M.; Costa, F.; Cunliffe, D.; Dreibelbis, R.; Eisenberg, J. N. S.; Evans, B.; Girones, R.; Hrudey, S.; Willetts, J.; Wright, C. Y. COVID-19: Urgent Actions, Critical Reflections and Future Relevance of 'WaSH': Lessons for the Current and Future Pandemics. J. Water Health 2020, 18, 613.

(4) Yates, T.; Vujcic, J. A.; Joseph, M. L.; Gallandat, K.; Lantagne, D. Water, Sanitation, and Hygiene Interventions in Outbreak Response: A Synthesis of Evidence. Waterlines 2018, 37 (1), 5-30. 
(5) WHO. Water, Sanitation, Hygiene, and Waste Management for SARS-CoV-2, the Virus That Causes COVID-19: Interim Guidance. WHO/2019-nCoV/IPC_WASH/2020.4; World Health Organization, 2020.

(6) CDC. Cleaning and Disinfecting Your Home: Everyday Steps and Extra Steps When Someone is Sick. https://www.cdc.gov/ coronavirus/2019-ncov/prevent-getting-sick/disinfecting-your-home. html (accessed 2021-03-10).

(7) Bedrosian, N.; Mitchell, E.; Rohm, E.; Rothe, M.; Kelly, C.; String, G.; Lantagne, D. A Systematic Review of Surface Contamination, Stability, and Disinfection Data on SARS-CoV-2 (Through July 10, 2020). Environ. Sci. Technol. 2021, 55, 4162.

(8) Sinclair, R. G.; Rose, J. B.; Hashsham, S. A.; Gerba, C. P.; Haas, C. N. Criteria for Selection of Surrogates Used To Study the Fate and Control of Pathogens in the Environment. Appl. Environ. Microbiol. 2012, 78 (6), 1969-1977.

(9) Nicklas, W.; Bleich, A.; Mähler, M. Chapter 3.2 - Viral Infections of Laboratory Mice. In The Laboratory Mouse, 2nd ed.; Hedrich, H. J., Ed.; Academic Press: Boston, 2012; pp 427-480.

(10) Hulkower, R. L.; Casanova, L. M.; Rutala, W. A.; Weber, D. J.; Sobsey, M. D. Inactivation of Surrogate Coronaviruses on Hard Surfaces by Health Care Germicides. Am. J. Infect. Control 2011, 39 (5), 401-407.

(11) Chiem, K.; Morales Vasquez, D.; Park, J.-G.; Platt, R. N.; Anderson, T.; Walter, M. R.; Kobie, J. J.; Ye, C.; Martinez-Sobrido, L. Generation and Characterization of Recombinant SARS-CoV-2 Expressing Reporter Genes. J. Virol. 2021, 95 (7), No. e02209-20.

(12) Xie, X.; Muruato, A. E.; Zhang, X.; Lokugamage, K. G.; FontesGarfias, C. R.; Zou, J.; Liu, J.; Ren, P.; Balakrishnan, M.; Cihlar, T.; Tseng, C.-T. K.; Makino, S.; Menachery, V. D.; Bilello, J. P.; Shi, P.-Y. A Nanoluciferase SARS-CoV-2 for Rapid Neutralization Testing and Screening of Anti-Infective Drugs for COVID-19. Nat. Commun. 2020, 11, 5214 .

(13) Aquino de Carvalho, N.; Stachler, E. N.; Cimabue, N.; Bibby, K. Evaluation of Phi6 Persistence and Suitability as an Enveloped Virus Surrogate. Environ. Sci. Technol. 2017, 51 (15), 8692-8700.

(14) Valegård, K.; Liljas, L.; Fridborg, K.; Unge, T. The ThreeDimensional Structure of the Bacterial Virus MS2. Nature 1990, 345 (6270), 36-41.

(15) Silverman, A. I.; Boehm, A. B. Systematic Review and MetaAnalysis of the Persistence and Disinfection of Human Coronaviruses and Their Viral Surrogates in Water and Wastewater. Environ. Sci. Technol. Lett. 2020, 7, 544 .

(16) Adcock, N. J.; Rice, E. W.; Sivaganesan, M.; Brown, J. D.; Stallknecht, D. E.; Swayne, D. E. The Use of Bacteriophages of the Family Cystoviridae as Surrogates for H5N1 Highly Pathogenic Avian Influenza Viruses in Persistence and Inactivation Studies. J. Environ. Sci. Health, Part A: Toxic/Hazard. Subst. Environ. Eng. 2009, 44 (13), $1362-1366$

(17) Gallandat, K.; Lantagne, D. Selection of a Biosafety Level 1 (BSL-1) Surrogate to Evaluate Surface Disinfection Efficacy in Ebola Outbreaks: Comparison of Four Bacteriophages. PLoS One 2017, 12 (5), No. e0177943.

(18) Morin, T.; Martin, H.; Soumet, C.; Fresnel, R.; Lamaudière, S.; Le Sauvage, A. L.; Deleurme, K.; Maris, P. Comparison of the Virucidal Efficacy of Peracetic Acid, Potassium Monopersulphate and Sodium Hypochlorite on Bacteriophages P001 and MS2. J. Appl. Microbiol. 2015, 119 (3), 655-665.

(19) Julian, T. R.; Trumble, J. M.; Schwab, K. J. Evaluating Efficacy of Field-Generated Electrochemical Oxidants on Disinfection of Fomites Using Bacteriophage MS2 and Mouse Norovirus MNV-1 as Pathogenic Virus Surrogates. Food Environ. Virol. 2014, 6 (2), 145155.

(20) String, G. M.; Gutiérrez, E. V.; Lantagne, D. S. Laboratory Efficacy of Surface Disinfection Using Chlorine against Vibrio Cholerae. J. Water Health 2020, 18 (6), 1009-1019.

(21) Grass, G.; Rensing, C.; Solioz, M. Metallic Copper as an Antimicrobial Surface. Appl. Environ. Microbiol. 2011, 77 (5), 15411547.
(22) Slayton, R. B.; Murphy, J. L.; Morris, J.; Faith, S. H.; Oremo, J.; Odhiambo, A.; Ayers, T.; Feinman, S. J.; Brown, A. C.; Quick, R. E. A Cluster Randomized Controlled Evaluation of the Health Impact of a Novel Antimicrobial Hand Towel on the Health of Children Under 2 Years Old in Rural Communities in Nyanza Province, Kenya. Am. J. Trop. Med. Hyg. 2016, 94 (2), 437-444.

(23) Iqbal, Q.; Lubeck-Schricker, M.; Wells, E.; Wolfe, M. K.; Lantagne, D. Shelf-Life of Chlorine Solutions Recommended in Ebola Virus Disease Response. PLoS One 2016, 11 (5), No. e0156136.

(24) Huang, J.; Hume, A. J.; Abo, K. M.; Werder, R. B.; VillacortaMartin, C.; Alysandratos, K.-D.; Beermann, M. L.; Simone-Roach, C.; Lindstrom-Vautrin, J.; Olejnik, J.; Suder, E. L.; Bullitt, E.; Hinds, A.; Sharma, A.; Bosmann, M.; Wang, R.; Hawkins, F.; Burks, E. J.; Saeed, M.; Wilson, A. A.; Mühlberger, E.; Kotton, D. N. SARS-CoV-2 Infection of Pluripotent Stem Cell-Derived Human Lung Alveolar Type 2 Cells Elicits a Rapid Epithelial-Intrinsic Inflammatory Response. Cell Stem Cell 2020, 27 (6), 962-973.e7.

(25) Adams, M. Bacteriophages; Interscience Publishers division of John Wiley \& Sons, Inc.: New York, NY, 1959.

(26) ASTM E35 Committee. Quantitative Disk Carrier Test Method for Determining Bactericidal, Virucidal, Fungicidal, Mycobactericidal, and Sporicidal Activities of Chemicals. ASTM International.

(27) U.S. EPA. About List N: Disinfectants for Coronavirus (COVID-19). https://www.epa.gov/pesticide-registration/list-ndisinfectants-coronavirus-covid-19 (accessed 2021-03-10).

(28) Xiling, G.; Yin, C.; Ling, W.; Xiaosong, W.; Jingjing, F.; Fang, L.; Xiaoyan, Z.; Yiyue, G.; Ying, C.; Lunbiao, C.; Liubo, Z.; Hong, S.; Yan, X. In Vitro Inactivation of SARS-CoV-2 by Commonly Used Disinfection Products and Methods. Sci. Rep. 2021, 11 (1), 2418.

(29) Sterk, E. Filovirus Haemorrhagic Fever Guideline; Médecins Sans Frontières, 2008; p 134.

(30) Hoffmann, M.; Kleine-Weber, H.; Schroeder, S.; Krüger, N.; Herrler, T.; Erichsen, S.; Schiergens, T. S.; Herrler, G.; Wu, N.-H.; Nitsche, A.; Müller, M. A.; Drosten, C.; Pöhlmann, S. SARS-CoV-2 Cell Entry Depends on ACE2 and TMPRSS2 and Is Blocked by a Clinically Proven Protease Inhibitor. Cell 2020, 181 (2), 271-280.e8.

(31) Nédellec, P.; Dveksler, G. S.; Daniels, E.; Turbide, C.; Chow, B.; Basile, A. A.; Holmes, K. V.; Beauchemin, N. Bgp2, a New Member of the Carcinoembryonic Antigen-Related Gene Family, Encodes an Alternative Receptor for Mouse Hepatitis Viruses. J. Virol. 1994, 68 (7), 4525-4537.

(32) Peng, G.; Sun, D.; Rajashankar, K. R.; Qian, Z.; Holmes, K. V.; Li, F. Crystal Structure of Mouse Coronavirus Receptor-Binding Domain Complexed with Its Murine Receptor. Proc. Natl. Acad. Sci. U. S. A. 2011, 108 (26), 10696-10701.

(33) Lan, J.; Ge, J.; Yu, J.; Shan, S.; Zhou, H.; Fan, S.; Zhang, Q.; Shi, X.; Wang, Q.; Zhang, L.; Wang, X. Structure of the SARS-CoV-2 Spike Receptor-Binding Domain Bound to the ACE2 Receptor. Nature 2020, 581 (7807), 215-220.

(34) Gallandat, K.; Wolfe, M. K.; Lantagne, D. Surface Cleaning and Disinfection: Efficacy Assessment of Four Chlorine Types Using Escherichia Coli and the Ebola Surrogate Phi6. Environ. Sci. Technol. 2017, 51 (8), 4624-4631.

(35) Wood, J. P.; Richter, W.; Sunderman, M.; Calfee, M. W.; Serre, S.; Mickelsen, L. Evaluating the Environmental Persistence and Inactivation of MS2 Bacteriophage and the Presumed Ebola Virus Surrogate Phi6 Using Low Concentration Hydrogen Peroxide Vapor. Environ. Sci. Technol. 2020, 54 (6), 3581-3590.

(36) Brown, T. Inactivation of Bacteriophage $\Phi 6$ on Tyvek Suit Surfaces by Chemical Disinfection. MPH Thesis, Georgia State University, Atlanta, 2015.

(37) Tseng, C.-C.; Li, C.-S. Inactivation of Viruses on Surfaces by Ultraviolet Germicidal Irradiation. J. Occup. Environ. Hyg. 2007, 4 (6), 400-405.

(38) Vatter, P.; Hoenes, K.; Hessling, M. Photoinactivation of the Coronavirus Surrogate Phi6 by Visible Light. Photochem. Photobiol. 2021, 97 (1), 122-125.

(39) Tuladhar, E.; Hazeleger, W. C.; Koopmans, M.; Zwietering, M. H.; Beumer, R. R.; Duizer, E. Residual Viral and Bacterial 
Contamination of Surfaces after Cleaning and Disinfection. Appl. Environ. Microbiol. 2012, 78 (21), 7769-7775.

(40) Baek, S.-B.; Kim, S.-W.; Ha, S.-D. Reduction of Escherichia Coli on Surfaces of Utensils and Development of a Predictive Model as a Function of Concentration and Exposure Time of Chlorine. Foodborne Pathog. Dis. 2012, 9 (1), 1-6.

(41) Lombardi, M. E.; Ladman, B. S.; Alphin, R. L.; Benson, E. R. Inactivation of Avian Influenza Virus Using Common Detergents and Chemicals. Avian Dis. 2008, 52 (1), 118-123.

(42) Borkow, G.; Gabbay, J. Putting Copper into Action: CopperImpregnated Products with Potent Biocidal Activities. FASEB J. 2004, 18 (14), 1728-1730.

(43) Mantlo, E.; Paessler, S.; Seregin, A. V.; Mitchell, A. T. LuminoreCopperTouch ${ }^{\mathrm{TM}}$ Surface Coating Effectively Inactivates SARS-CoV-2, Ebola and Marburg Viruses in Vitro. medRxiv 2020, DOI: $10.1101 / 2020.07 .05 .20146043$.

(44) van Doremalen, N.; Bushmaker, T.; Morris, D. H.; Holbrook, M. G.; Gamble, A.; Williamson, B. N.; Tamin, A.; Harcourt, J. L.; Thornburg, N. J.; Gerber, S. I.; Lloyd-Smith, J. O.; de Wit, E.; Munster, V. J. Aerosol and Surface Stability of SARS-CoV-2 as Compared with SARS-CoV-1. N. Engl. J. Med. 2020, 382, 1564.

(45) Torondel, B.; Khan, R.; Holm Larsen, T.; White, S. Efficacy of the SuperTowel®: An Alternative Hand-Washing Product for Humanitarian Emergencies. Am. J. Trop. Med. Hyg. 2019, 100 (5), $1278-1284$.

(46) Bloomfield, S. F.; Arthur, M.; Van Klingeren, B.; Pullen, W.; Holah, J. T.; Elton, R. An Evaluation of the Repeatability and Reproducibility of a Surface Test for the Activity of Disinfectants. J. Appl. Bacteriol. 1994, 76 (1), 86-94.

(47) Prussin, A. J.; Schwake, D. O.; Lin, K.; Gallagher, D. L.; Buttling, L.; Marr, L. C. Survival of the Enveloped Virus Phi6 in Droplets as a Function of Relative Humidity, Absolute Humidity, and Temperature. Appl. Environ. Microbiol. 2018, 84 (12), e00551-18 DOI: 10.1128/AEM.00551-18.

(48) Wigginton, K. R.; Pecson, B. M.; Sigstam, T.; Bosshard, F.; Kohn, T. Virus Inactivation Mechanisms: Impact of Disinfectants on Virus Function and Structural Integrity. Environ. Sci. Technol. 2012, 46 (21), 12069-12078. 